Artigo

\title{
Variabilidad de Baja Frecuencia de la Persistencia de la Temperatura en el Sudeste de Sudamérica
}

\author{
Gustavo Naumann ${ }^{1,2}$, Walter Vargas ${ }^{1,2}$ \\ ${ }^{1}$ Consejo Nacional de Investigaciones Cientificas y Técnicas, Buenos Aires, Argentina. \\ ${ }^{2}$ Departamento de Ciencias de la Atmósfera y los Océanos. Universidad de Buenos Aires, \\ Buenos Aires, Argentina.
}

Recebido em 21 de Abril de 2015 - Aceito em 3 de Junho de 2016

\begin{abstract}
Resumen
Se analizan las estructuras de las autocovarianzas y autocorrelaciones de las temperaturas máximas y mínimas en estaciones de referencia en el sudeste de Sudamérica. A través de estas propiedades se observa que existen oscilaciones de períodos entre 18 y 25 años en las series bajo estudio, aunque estas periodicidades presentan cambios con el tiempo, especialmente entre los años 1950-1970. La excepción es Río Gallegos donde la periodicidad de 18 años se observa durante todo el registro. La persistencia definida mediante el primer coeficiente de autocorrelación es función de la cantidad de términos de autocovarianza negativas. No obstante, durante algunos años es dependiente de la magnitud de los términos positivos dados por las irrupciones cálidas o frías intensas. Entre los años 1950 y 1970 se ha observado un aumento de la variabilidad con una alta alternancia entre períodos de mayor y menor persistencia. Estos cambios analizados en la persistencia sugieren una variación en la frecuencia de distintos tipos de circulación que tienen un impacto directo en la estructura térmica regional. Así mismo, variaciones de este tipo pueden tener serios impactos socio-económicos ya que estos afectarían directamente la frecuencia y duración de los eventos extremos. Esto tiene importancia también en el diseño de métodos de control y consistencia ya que en ellos es posible considerar como outlier o información errónea los cambios producidos por las irrupciones de escala sinóptica mencionadas anteriormente.
\end{abstract}

Palabras-clave: variabilidad climática, memoria, temperatura diaria, análisis wavelet.

\section{Low-Frequency Variability of Surface Air Temperature Persistence in Southern South America}

\begin{abstract}
Eight reference stations in south-eastern South America are analysed to derive the auto-covariance and auto-correlations of maximum and minimum temperature. Through the analysis are observed oscillations of periods between 18 and 25 years in the series studied. These periodicities show changes over time, especially in the 1950-1970 period. The exception is Río Gallegos where a periodicity of 18 years is observed throughout the entire record. Persistence is defined as the first correlation coefficient and is a function of the number of negative terms in the auto-covariance. However for some years it is mainly driven by the magnitude of the positive terms given by extreme warm or cold outbreaks. Between 1950 and 1970 it was observed an increased variability in the analysed properties. These changes suggest a variation in the frequency of different circulation patterns having a direct impact on the regional thermal structure. Likewise, this kind of variations can have serious socio-economic impacts as these directly affect the frequency and duration of extreme events. This is relevant in the design of consistency and quality control methods to detect outliers or systematic errors.

Keywords: climate variability, memory, daily temperature, wavelet analysis.
\end{abstract}

\section{Introduccion}

La variabilidad climática en la atmósfera se origina a través de la presencia de inestabilidad de diversos tipos que, particularmente, produce variabilidad de alta frecuencia conocida usualmente como fenómenos sinópticos (tiempo, Hasselmann, 1976). El tiempo se refiere al comportamiento

Autor de correspondência: Gustavo Naumann, gnaumann@at.fcen.uba.ar. 
de la atmósfera sobre períodos de varios días. Mientras que por otro lado, el clima trata generalmente sobre el comportamiento medio del sistema o procesos de baja frecuencia, que se encuentran condicionados por procesos físicos asociados a las condiciones de borde de la atmósfera. Para caracterizar los procesos climáticos es necesario describir el comportamiento a largo plazo de las variables analizadas y usualmente se define a partir de períodos mayores a 30 años.

Por la amplitud de los eventos de alta frecuencia $\left(f<1 / 30\right.$ dias $\left.^{-1}\right)$ un estado climático está sujeto a fluctuaciones estocásticas en general impredecibles en las escalas temporales de interés climático (Trenberth, 1984b, Madden y Shea, 1978). Sin embargo, una parte de la variabilidad natural asociada con la evolución de ondas de baja frecuencia $\left(f \geq 1 / 30\right.$ días $\left.^{-1}\right)$ puede mejorar la predictibilidad del sistema (Shukla, 1998). La comprensión de la variabilidad de baja frecuencia es fundamental para extender el rango de la predicción numérica de la atmósfera.

Sin embargo, la principal dificultad que presenta el sistema climático es el carácter transitivo de su evolución. En la escala diaria, la evolución del tiempo esta caracterizada por abruptos cambios definidos por diversos patrones de circulación. Esto permite caracterizar las fluctuaciones del clima en una región por las transiciones entre éstos tipos de circulación. Algunas investigaciones en la región muestran que las series de tiempo registradas sobre las temperaturas extremas diarias contienen suficiente información para describir procesos de mayor escala debidos a cambios en la circulación atmosférica (Vargas y Naumann, 2008; Naumann y Vargas, 2009).

Para describir estos procesos sobre la temperatura diaria en función de estas asociaciones y como primera aproximación a modelos de predicción independientes del pronosticador (pronósticos objetivos), la temperatura puede ser aproximada por un proceso Markoviano. Usualmente un modelo de Markov se refiere a un proceso estocástico discreto en el que la probabilidad de que ocurra un evento depende de los eventos anteriores (Gabriel and Neumann, 1962). Esto significa que dado un estado $x_{t}$ para el tiempo $t$, se mantiene inalterada la probabilidad condicional de un estado futuro en el tiempo $t+\tau$, aunque se introduzca información de tiempos anteriores a $t$. La distribución de estados futuros $x_{t+} \tau$ usualmente se denomina como probabilidades de transición (Wilks, 2002). Las probabilidades de transición también implican un grado específico de correlación serial o persistencia. Por esto, es posible escribir las probabilidades de transición en términos del primer coeficiente de autocorrelacion $\left(r_{1}\right.$ para un desfase de 1 paso de tiempo). En el contexto de una cadena de Markov, $r_{1}$ es conocido usualmente como el parámetro que define la persistencia (Wilks, 2002). Por otro lado, la antipersistencia se define como la probabilidad o habilidad del sistema a cambiar de estado. En este contexto se refiere a los términos negativos de autocovarianza de $r_{1}$.
En el sudeste de Sudamérica varios estudios analizaron el comportamiento de la temperatura y sus extremos. Rusticucci y Vargas (1995) caracterizaron la circulación de superficie en situaciones de temperatura extremas, olas de calor y de frío, desde el punto de vista climático sinóptico. Estudios climáticos sobre la ocurrencia de irrupciones extremas de masas de aire cálidas o frías en cualquier época del año, y su variabilidad interanual, se pueden encontrar en Rusticucci y Vargas $(1993,2001)$ y Tencer y Rusticucci (2012). En referencia a estudios sobre las temperaturas extremas en Argentina, Rusticucci y Barrucand (2001) analizaron la relación entre la temperatura media estacional y la ocurrencia de días extremos, mientras que Barrucand y Rusticucci (2001) efectuaron un estudio de la variabilidad temporal y regional de la frecuencia de eventos extremos en el país.

Sin embargo, pocos trabajos en la región se centran en el análisis de la persistencia y su potencial para describir la predictibilidad de la atmósfera. En este sentido Naumann et al. (2011) mostraron que existen cambios estacionales y espaciales en la persistencia, definida ésta, por los tres primeros coeficientes de la función de autocorrelacion. Además se ha observado una mayor variabilidad de la persistencia de la temperatura mínima y del tiempo de independencia (tiempo en días en el que las observaciones se vuelven independientes) durante invierno y primavera. Diferente comportamiento se observó para la temperatura máxima, con la mayor variabilidad durante el otoño. En particular las variaciones interanuales de la persistencia reflejan en cierta medida los procesos que conducen al principio o al final del otoño e invierno.

En este trabajo se desea establecer si además existe una variabilidad interanual definida que pueda producir cambios en la estimación de los modelos de diagnóstico y pronóstico objetivo, entendiéndose por éstos como aquéllos efectuados independientemente del pronosticador. Para esto se propone la estimación de la función de autocorrelacion sobre ocho estaciones centenarias en Argentina y el sur de Brasil para distintas submuestras de cada serie temporal utilizando dos metodologías distintas. En particular se consideró el cálculo de la función de autocorrelacion y autocovarianza para (i) diferentes submuestras conformadas por la información diaria de cada año del registro y (ii) considerando los valores medios para la muestra completa. Así mismo, se analizan las periodicidades sobre las series que definen la persistencia de la temperatura mediante el análisis espectral wavelet.

\section{Datos y Metodos}

\subsection{Base de datos}

Para el análisis se seleccionaron ocho estaciones en el sudeste de Sudamérica con registros de temperatura máxima y mínima en escala diaria (detalles en Tabla 1 y Fig. 1). En particular, se seleccionaron estas localidades por la 
Tabla 1 - Descripción, ubicación geográfica, período de observaciones y porcentaje de datos faltantes para las ocho estaciones de referencia analizadas.

\begin{tabular}{|c|c|c|c|c|c|c|}
\hline País & Estación & Longitud & Latitud & Inicio & Fin & $\%$ faltantes \\
\hline \multirow[t]{7}{*}{ Argentina } & Santa Rosa & -64.26 & -36.54 & 1937 & 2007 & 2 \\
\hline & Río Gallegos & -69.45 & -51.99 & 1896 & 2007 & 10 \\
\hline & Pergamino & -60.53 & -33.90 & 1931 & 2007 & 1 \\
\hline & Corrientes & -58.74 & -27.43 & 1894 & 2007 & 6 \\
\hline & Pilar & -63.85 & -31.64 & 1931 & 2007 & 3 \\
\hline & S. M. Tucumán & -65.20 & -26.80 & 1891 & 2007 & 0 \\
\hline & Buenos Aires & -58.42 & -34.57 & 1906 & 2007 & 0 \\
\hline Brasil & Campinas & -47.12 & -23.00 & 1890 & 2003 & 1 \\
\hline
\end{tabular}

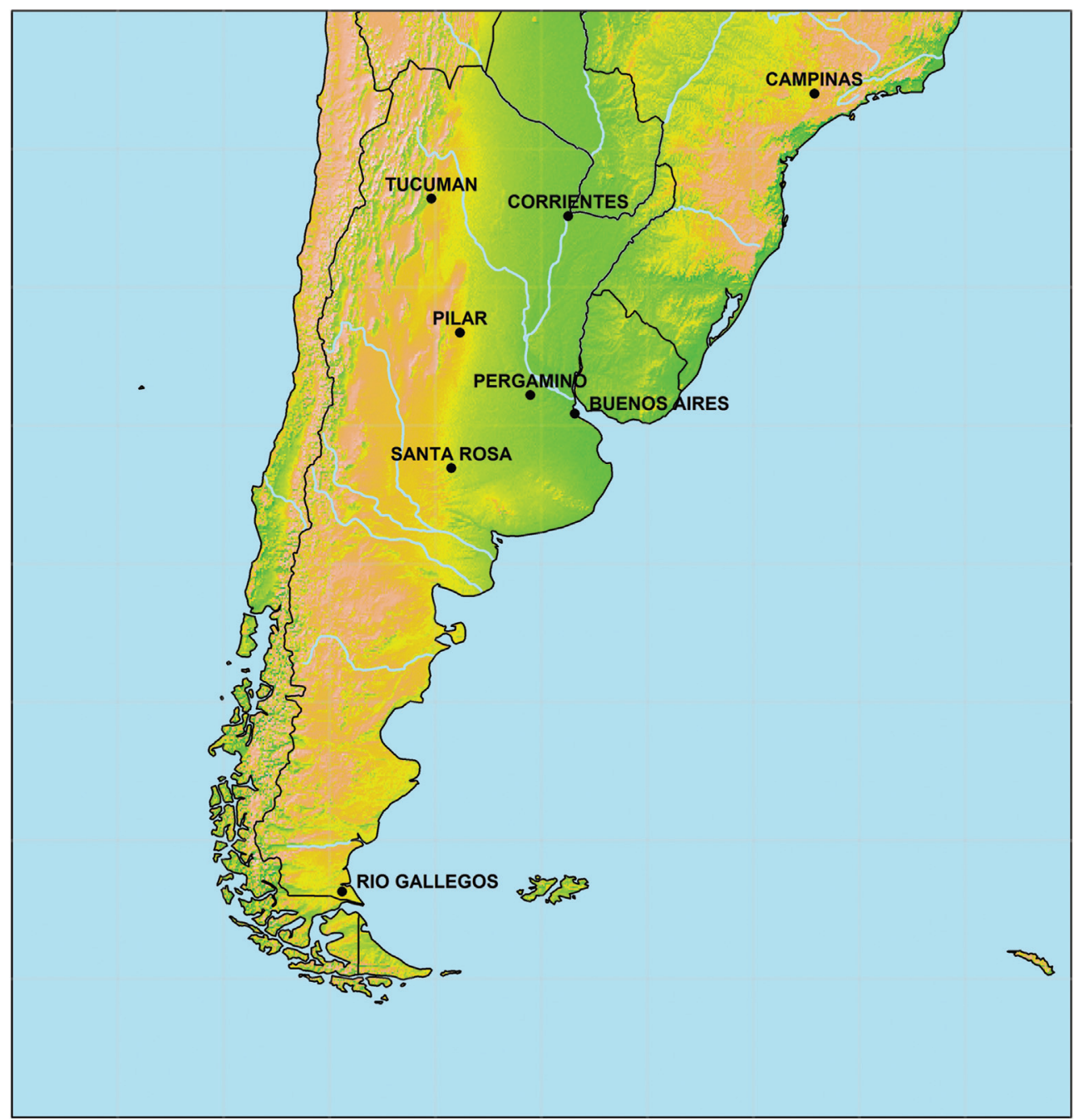

Figura 1 - Localización geográfica de las estaciones utilizadas en el presente estudio.

longitud de sus registros (mayor a 70 años) de información diaria. Conjuntamente, la distribución geográfica de estas estaciones se considera representativa del área de estudio, describiendo las regiones climáticas del Sudeste de Sudamérica, cubriendo una amplia sección latitudinal (Fig. 1).

Antes de realizar el estudio, se efectuó un análisis de consistencia básica en las series diarias de temperatura máxima y mínima siguiendo, entre otras, las recomendaciones de la guía de control de calidad de datos climatológicos de superficie (WMO, 2002).

Cabe destacar que se han detectado algunas inhomogeneidades sobre las series de Corrientes y Río Gallegos debido a la relocalización de las estaciones, mientras que en Campinas y Buenos Aires el crecimiento urbano impuso un 
calentamiento antropogénico sostenido durante el período analizado. Las series analizadas ya han sido utilizadas en Vargas y Naumann (2008), donde se detalla la metodología en que ambos tipos de inhomogeneidades fueron corregidas siguiendo un análisis de consistencia interna y detección de inhomogeneidades. En particular se detectaron cambios en la temperatura debidos a la relocalización de las estaciones en Corrientes y Río Gallegos y el crecimiento urbano en Buenos Aires y Campinas. Luego de la consistencia inicial se cuantificó la presencia de datos faltantes (Tabla 1). Dado que los valores analizados en este estudio son anuales derivados de información diaria se calcularon los coeficientes de autocorrelación y autocovarianzas solo para los años que registraron más del 75\% de información.

\subsection{Estimación de la función de autocorrelación}

Las autocovarianzas muestrales para el desfase $k, C_{k}$ (Wilks, 2002), se pueden estimar como;

$$
C_{k}=\frac{1}{N} \sum_{i=k+1}^{N}\left(x_{i+k}-\bar{x}\right)\left(x_{i}-\bar{x}\right)
$$

donde $\bar{x}$ es la media de una muestra con $N$ datos que representan el total de años por localidad. Además $C_{0}$ representa la varianza total de la muestra,

$$
C_{0}=S_{x}=\frac{1}{N} \sum_{i=1}^{N}\left(x_{i}-\bar{x}\right)^{2}
$$

Con estos elementos, el coeficiente de autocorrelación para el desfase $k$ se puede definir como;

$$
r_{k}=\frac{C_{k}}{C_{0}}
$$

Según la definición de la Ec. (3) se obtiene la función de autocorrelación a nivel climático (donde se considera la media para todo el período). Sin embargo, para muchas aplicaciones es de interés conocer el comportamiento entre las asociaciones para distintos períodos o sub-muestras según sea el problema que se desea tratar.

Por ejemplo, si se desea analizar la variabilidad interanual de la función de autocorrelación es posible dividir la serie en muestras de $N$ observaciones para $J$ años. A partir de esto, se proponen dos posibles aproximaciones para estimar las autocovarianzas.

Si $x_{i j}$ es el $i$-ésimo día para el año $j$, se define

$$
C_{k, j}=\frac{1}{N} \sum_{i=k+1}^{N}\left(x_{i+k, j}-\bar{x}_{j}\right)\left(x_{i, j}-\bar{x}_{j}\right)
$$

donde

$\bar{x}_{j}=\frac{1}{N} \sum_{i=1}^{N} x_{i, j}$

es la media de la muestra para el año $j$. A partir de estos elementos, y siguiendo el planteo desarrollado en
Trenbertth, (1984a) es posible definir el coeficiente de autocorrelación en el desfase $k$ del año $j$, como

$$
\begin{aligned}
& r_{k, j}=\frac{C_{k, j}}{C_{0, j}} \\
& \mathrm{y} \\
& r_{k}=\frac{1}{J} \sum_{J=1}^{J} r_{k, j}
\end{aligned}
$$

es la autocorrelación total.

De acuerdo a Trenberth, 1984a, otra forma posible de definir la autocorrelación es

$$
C_{k, j}=\frac{1}{N} \sum_{i=k+1}^{N}\left(x_{i+k, j}-\bar{x}\right)\left(x_{i, j}-\bar{x}\right)
$$

donde

$$
\bar{x}=\frac{1}{N J} \sum_{j=1}^{J} \sum_{i=1}^{N} x_{i, j}
$$

es la media sobre todas las observaciones. Por consiguiente la autocovarianza total es,

$$
C_{k}=\frac{1}{J} \sum_{j=1}^{J} C_{k, j}
$$

y la autocorrelación

$$
r_{k}=\frac{C_{k}}{C_{0}}
$$

La principal diferencia entre estas estimaciones es el uso de medias para cada muestra (Ec. (4)), que permite comparar la variabilidad interna anual de la persistencia, mientras que según la Ec. (8) se utiliza la media total para referir los resultados anuales a un nivel común.

En la aplicación, la diferencia de ambos métodos permite apreciar contrastes en la forma de cuantificar la cantidad de perturbaciones que afectan el clima de una determinada región.

Si se analizan las desviaciones observadas respecto a cada año, es posible aproximar en mejor medida la variabilidad interna de la temperatura para este período específico. Mientras que con el segundo método es posible describir cuando las perturbaciones de un año específico resultan más o menos persistentes en relación a un único sistema de referencia que viene representado por la climatología.

Por ejemplo, para un año que se ha observado más cálido que la media climática, considerando el primer método se podrá estimar en mejor grado las transiciones entre estados del sistema, mientras que con el segundo método, será más probable la ocurrencia de anomalías positivas y por consiguiente una menor cantidad de cambios de estado, sin embargo con la segunda estimación es posible comparar 
la persistencia de este año con los del resto del período ya que todos son calculados respecto a una misma referencia.

\subsection{Estimación espectral wavelet}

El análisis espectral wavelet provee una forma de representar la variación de periodicidades con el tiempo, siendo una poderosa herramienta matemática que provee una representación en tiempo-frecuencia de una señal analizada en el dominio del tiempo (Percival y Walden, 2000). A partir de esta metodología no sólo es posible determinar valores particulares de frecuencias en series no estacionarias, sino también se puede determinar el tiempo en que ocurren estos cambios. Una completa descripción sobre la teoría del análisis wavelet se encuentra en Daubechies (1990), mientras que una completa descripción de la aplicación en geofísica puede ser encontrada en Torrence y Compo (1998).

En este trabajo se sigue la misma metodología detallada en Naumann y Vargas (2012) donde se ha efectuado un análisis wavelet sobre las mismas localidades utilizadas en este estudio para filtrar y describir la variabilidad intraestacional de la temperatura en la región.

Para realizar el análisis se utiliza una función wavelet que depende de un parámetro temporal adimensional. Para que una función sea admisible como wavelet, debe tener media igual a cero y estar localizada tanto en el espacio de tiempo como en el de frecuencia (Farge, 1992). En este trabajo se utilizó la función wavelet Morlet, que consiste en una onda plana modulada por una función Gaussiana. El nivel de significancia del espectro wavelet se determinó considerando un proceso de ruido rojo (a menor frecuencia, mayor poder).

\section{Resultados y Discusión}

En las Figs. 2 y 3 se muestran las marchas interanuales de $r_{1}$ (persistencia, en azul) y frecuencia anual de autocovarianzas negativas (antipersistencia, en rojo) estimadas sobre la temperatura máxima y mínima según las Ecs. (6) y (4), respectivamente, es decir cada autocovarianza es calculada respecto de los valores medios y varianzas de cada año.

La antipersistencia de la temperatura máxima observa un máximo en los años 1940-50 en Campinas, mientras que en Tucumán se observa un máximo relativo a fines de la década de 1930. Durante fines de la década de 1940 en Pilar se observa un mínimo relativo, mientras que en Santa Rosa la antipersistencia decrece durante la década de 1940 y alcanza un mínimo relativo a principios de 1950 . Así mismo, en Campinas se observa un máximo que comienza a mediados de la década de 1940; en Corrientes, Pilar y Santa Rosa el mínimo se observa a principios de la década de 1960; en Tucumán el mínimo está presente en la década de 1950 y a finales de 1960; mientras que en Pergamino el mínimo es a fines de 1950. La disminución (aumento) de la persistencia (antipersistencia) se relaciona con períodos de mayor variabilidad interna y disminución de la predictibilidad de corto plazo. Así mismo, un incremento en el desorden del sistema puede implicar un consiguiente cambio de estado (Naumann y Vargas, 2009). Remarcablemente estos dos períodos son coincidentes con dos saltos climáticos observados en muchas variables en la región (Minetti y Vargas, 1997).

Por otro lado, se observa que tanto para la temperatura máxima como la mínima los años con mayor (menor) persistencia están asociados a una menor (mayor) cantidad de términos negativos de la autocovarianza. Esto es particularmente evidente sobre las estaciones ubicadas en cercanías a los trópicos (Campinas, Tucumán y Corrientes). En la Tabla 2 se muestran las correlaciones entre $r_{1}$ y la frecuencia de autocovarianzas negativas donde se confirma el comportamiento inverso en las regiones tropicales. En todas estas estaciones se observan correlaciones negativas significativas excepto para la temperatura mínima en Corrientes. Lo mismo es válido para Buenos Aires y Río Gallegos.

A partir de esto es posible caracterizar los años dominados por fenómenos más persistentes que están asociados directamente a una disminución en la cantidad de cambios de estados. Sin embargo, a pesar de ser esto cierto, también se observan años donde hay una relación directa entre el primer coeficiente de autocorrelación y la frecuencia anual de autocovarianzas. Por ejemplo, en la temperatura mínima en Corrientes y Pilar durante finales de la década de 1950 y comienzos de la década de 1960 se registra un mínimo relativo de ambas variables. Esto implica que la cantidad de cambios de estado fue escasa durante este año, pero su alta intensidad es el factor que lleva a obtener un valor bajo de $r_{1}$.

Así mismo, los años donde la temperatura es altamente persistente (mayores valores de $r_{1}$ ), estos se asocian a pocos cambios de estado o estos son de baja amplitud. Estos años se caracterizan por la presencia de eventos cálidos o fríos extremos definidos por los valores que exceden el percentil 90 y debajo del percentil 10 de cada distribución respectivamente. La ocurrencia de estos eventos tiene una influencia indistinta sobre la persistencia dado que para ambos casos se tienen valores positivos de las contribuciones de la autocovarianza, que por su magnitud y persistencia influencian directamente la estimación del primer coeficiente de autocorrelación.

Si analizamos la variabilidad de baja frecuencia de estas series, en la Fig. 4 se muestra el espectro Wavelet asociado a las marchas de $r_{1}$ en función del tiempo para Campinas, Tucumán, Buenos Aires y Río Gallegos.

Considerando las cuatro localidades analizadas se observan escalas de tiempo características, desde la escala interanual hasta la decadal. Algunas bandas del espectro presentan características comunes en términos de frecuencias desde la escala interanual (fluctuaciones de entre 4-8 años) hasta escala decadal (12-20 años) y algunas de 

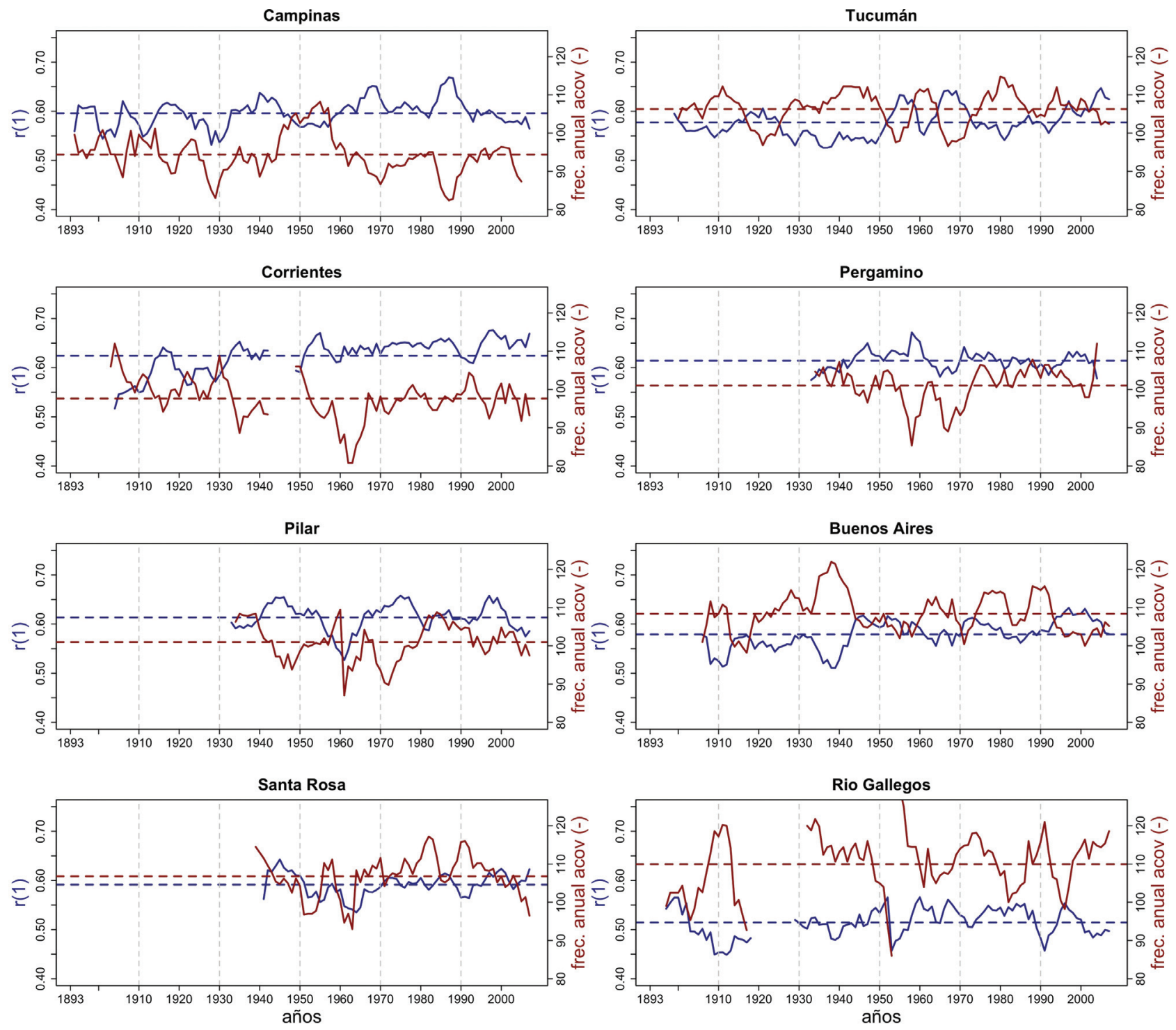

Figura 2 - Marcha interanual del primer coeficiente de autocorrelación calculado según Ec. (6) (azul), y valor medio (azul en línea de puntos) y frecuencia anual de autocovarianzas negativas para el desfase 1 (antipersistencia) en rojo (valor medio en línea roja de puntos) para la temperatura máxima en las estaciones de referencia.

largo plazo (32-64 años) aunque en el último caso los resultados se encuentran dentro del cono de incerteza.

En Campinas, Buenos Aires y Río Gallegos se observa una estructura común de la temperatura máxima en estas localidades en la forma de oscilaciones interdecadales durante todo el período de análisis. En Tucumán se observa una oscilación pluridecadal (32-64 años) durante todo el período, aunque este resultado no es conclusivo dado que esta banda de energía se encuentra dentro del área del "cono de influencia" donde los efectos de borde son significativos. En el espectro interanual Tucumán y Río Gallegos presentan oscilaciones significativas en períodos centrados en 12 años entre los años 1950-1970.

Para la temperatura mínima los períodos de máxima energía son más heterogéneos donde solamente Río Gallegos muestra un patrón de oscilación pluridecadal (12-28 años) durante todo el período y la aparición de un máximo centrado en la banda de 6 y 12 años durante el período 1960-1985. Estos cambios de baja frecuencia concuerdan con los resultados obtenidos en distintas variables del sistema climático por Compagnucci y Vargas (1983) para las precipitaciones estivales del noroeste argentino, Minetti y Carletto (1990) y Vargas et al. (2002), donde encuentran señales con períodos entre 17 y 26 años. Barrucand y Rusticucci (2001) detectan la presencia de ondas largas (mayores a 10 años) que dominan la variabilidad interanual de la frecuencia de días extremadamente fríos en el este del país (especialmente en verano), ondas medias (entre 3 y 10 años) en el centro y norte del país y ondas cortas (cuasi bienales, menores a 3 años) predominan en áreas costeras.

En las Figs. 5 y 6 se analizan las marchas interanuales de $r_{1}$ y frecuencia anual de autocovarianzas negativas esti- 

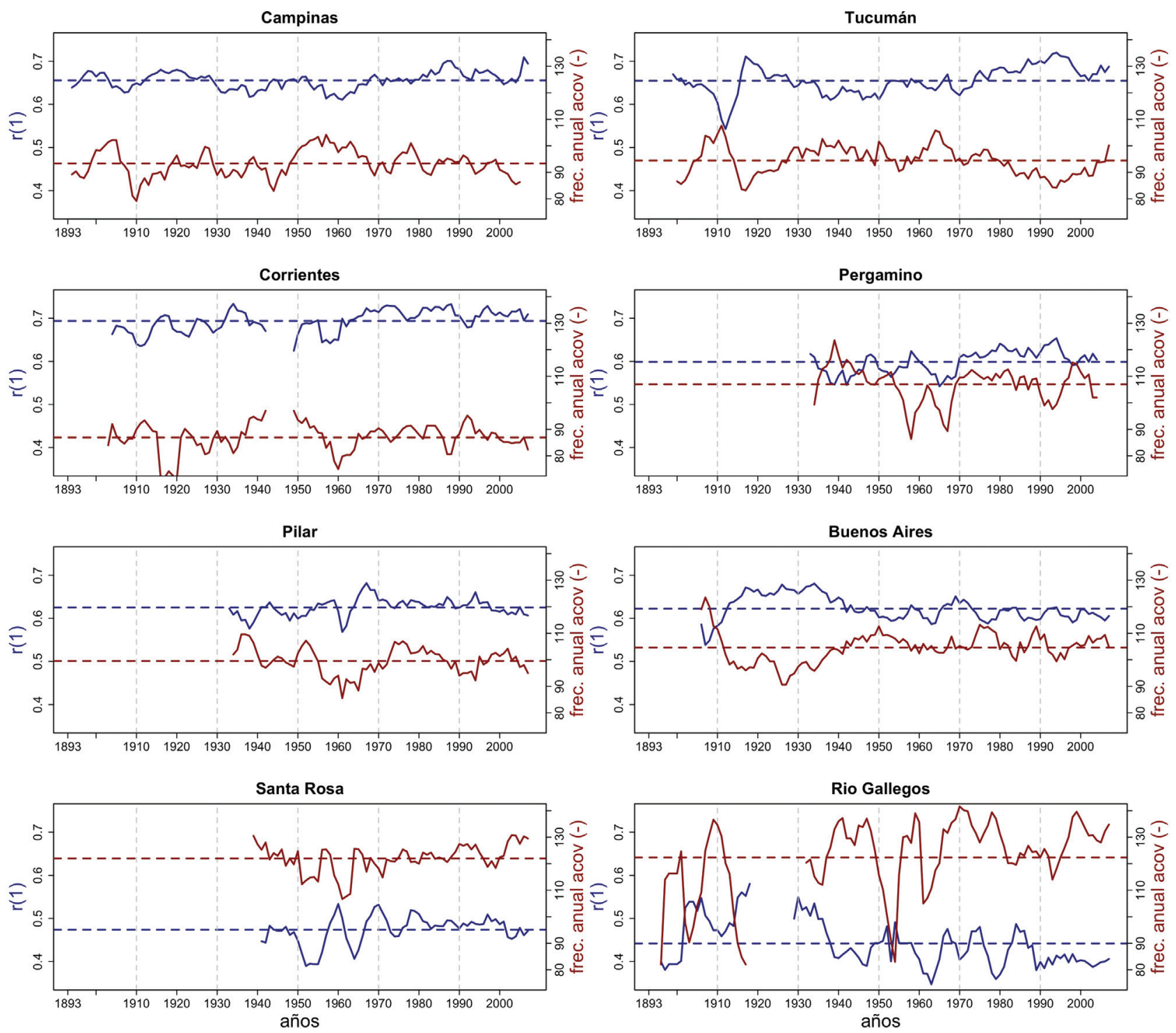

Figura 3 - Marcha interanual del primer coeficiente de autocorrelación calculado según Ec. (6) (azul), y valor medio (azul en línea de puntos) y frecuencia anual de autocovarianzas negativas para el desfase 1 (antipersistencia) en rojo (valor medio en línea de puntos roja) para la temperatura mínima en las estaciones de referencia.

Tabla 2 - Correlaciones entre el primer coeficiente de correlación $\left(r_{1}\right)$ y frecuencia de autocovarianzas negativas (antipersistencia) de la temperatura máxima $(T X)$ y mínima $(T N)$ de acuerdo a Ecs. (6)-(11).

\begin{tabular}{|c|c|c|c|c|}
\hline & \multicolumn{2}{|c|}{ Ec. (6) } & \multicolumn{2}{|c|}{ Ec. (11) } \\
\hline & $T X$ & $T N$ & $T X$ & $T N$ \\
\hline Campinas & -0.476 & -0.180 & -0.173 & -0.193 \\
\hline Tucuman & -0.670 & -0.698 & -0.402 & -0.236 \\
\hline Corrientes & -0.581 & -0.133 & -0.134 & 0.113 \\
\hline Pergamino & -0.413 & -0.171 & 0.118 & 0.356 \\
\hline Pilar & -0.208 & -0.257 & 0.156 & 0.309 \\
\hline Buenos Aires & -0.565 & -0.845 & -0.178 & -0.532 \\
\hline Santa Rosa & 0.080 & 0.045 & 0.218 & 0.047 \\
\hline Río Gallegos & -0.295 & -0.458 & 0.378 & -0.159 \\
\hline
\end{tabular}



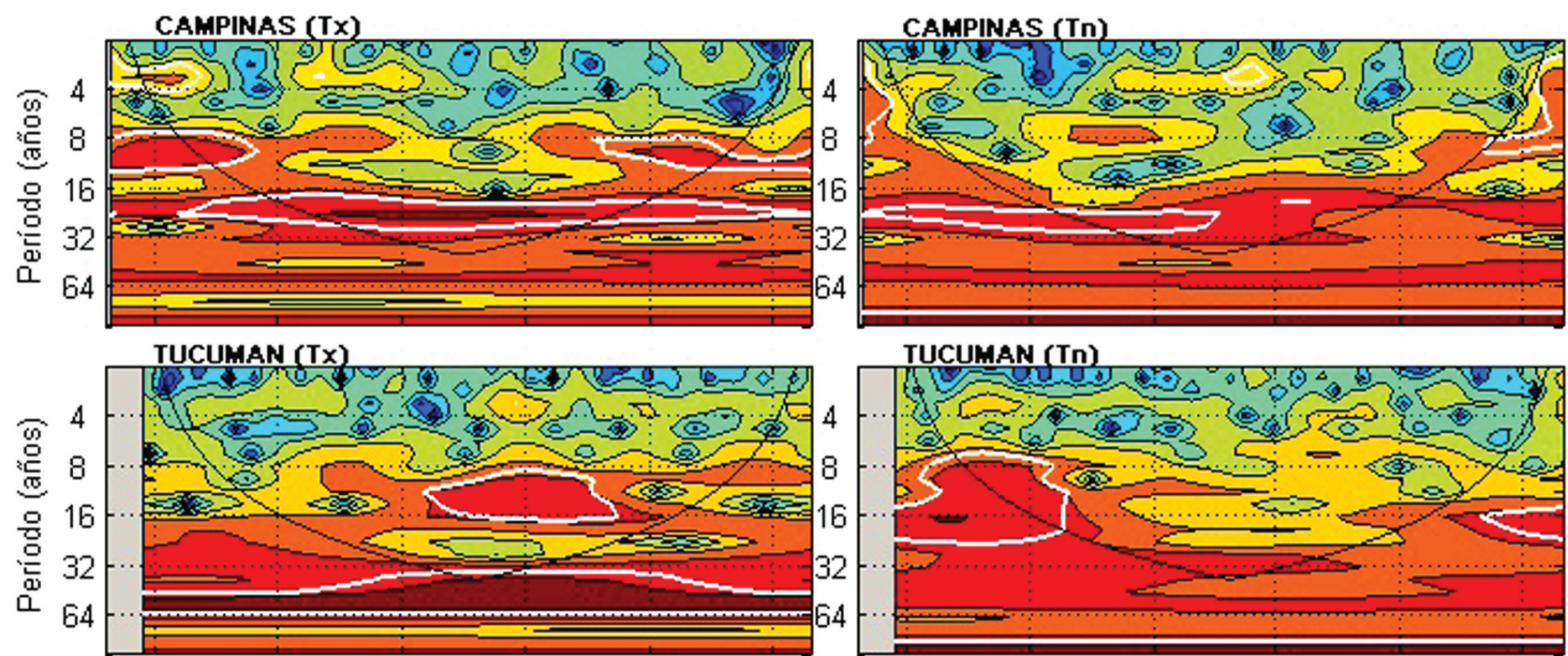

TUCUMAN (Tn)

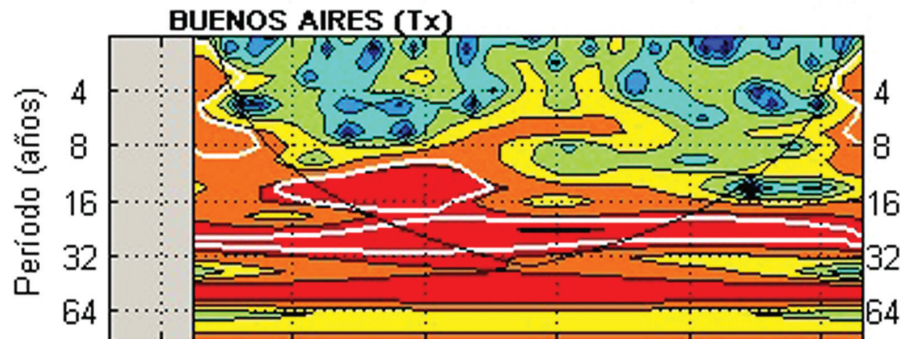

BUENOS AIRES (Tn)
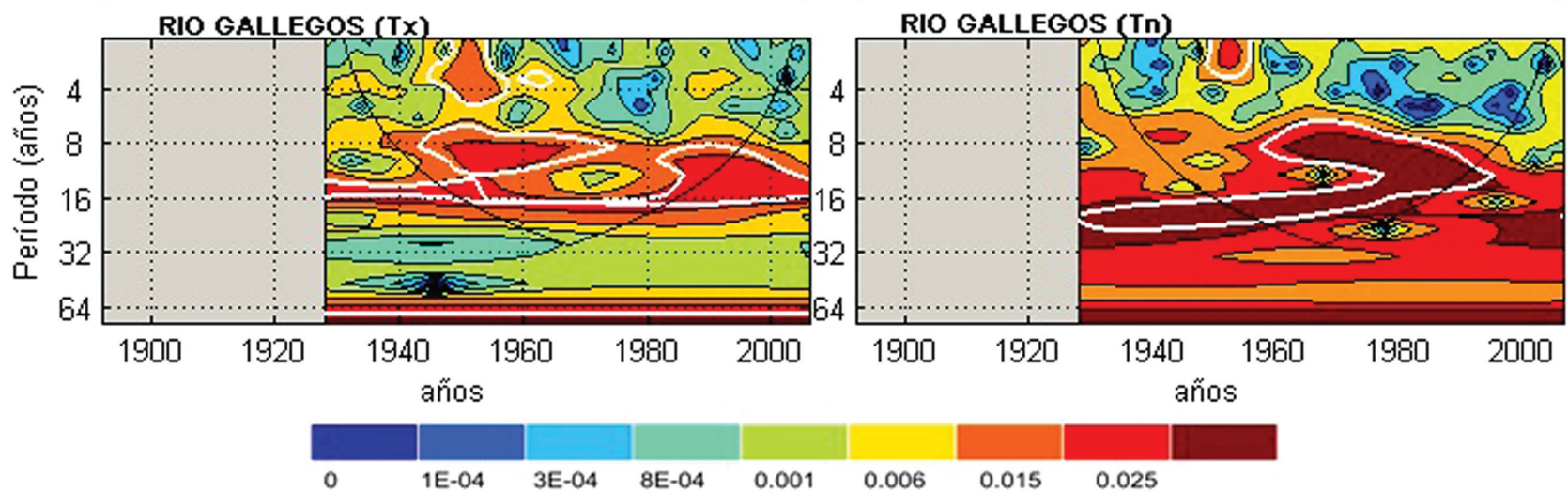

Figura 4 - Espectro Wavelet (adimensional) estimado en base a las marchas interanuales del primer coeficiente de autocorrelación calculado según Ec. (6). Los contornos blancos representan el nivel de significancia del $5 \%$, respecto de un modelo autoregresivo.

madas según Ecs. (8)-(11). En este caso cada autocovarianza es calculada respecto de los valores medios y varianzas de toda la muestra. Dado que cada año es comparado con los mismos parámetros de la muestra, esta estimación provee un sistema de referencia fijo, que permite analizar los cambios en el largo plazo.

En este caso y en contraste con el método anterior, la persistencia y antipersistencia parecen no estar acopladas salvo en Tucumán y la temperatura mínima en Buenos Aires, donde las correlaciones entre el primer coeficiente de correlación y frecuencia de autocovarianzas negativas son significativas (Tabla 2). Esto es producto de la menor variabilidad impuesta al $r_{1}$ al considerarse la media de la muestra en todos los casos. Por el otro lado, esta metodología (Figs. 5 y 6) permite detectar el mínimo observado sobre la antipersistencia en ambas temperaturas entre los años 1955-1965 en Corrientes, Pergamino, Pilar, Santa Rosa y Río Gallegos. Al igual que en el caso anterior, en estas representaciones se observa que las mayores variaciones se observan entre los años 1950-1970, en algunos casos con un cambio de la variabilidad (Campinas y Santa Rosa) y en otros en la forma de posibles saltos o cambios abruptos (Tucumán y Buenos Aires). Estos cambios se manifiestan en la disminución de la frecuencia de eventos 

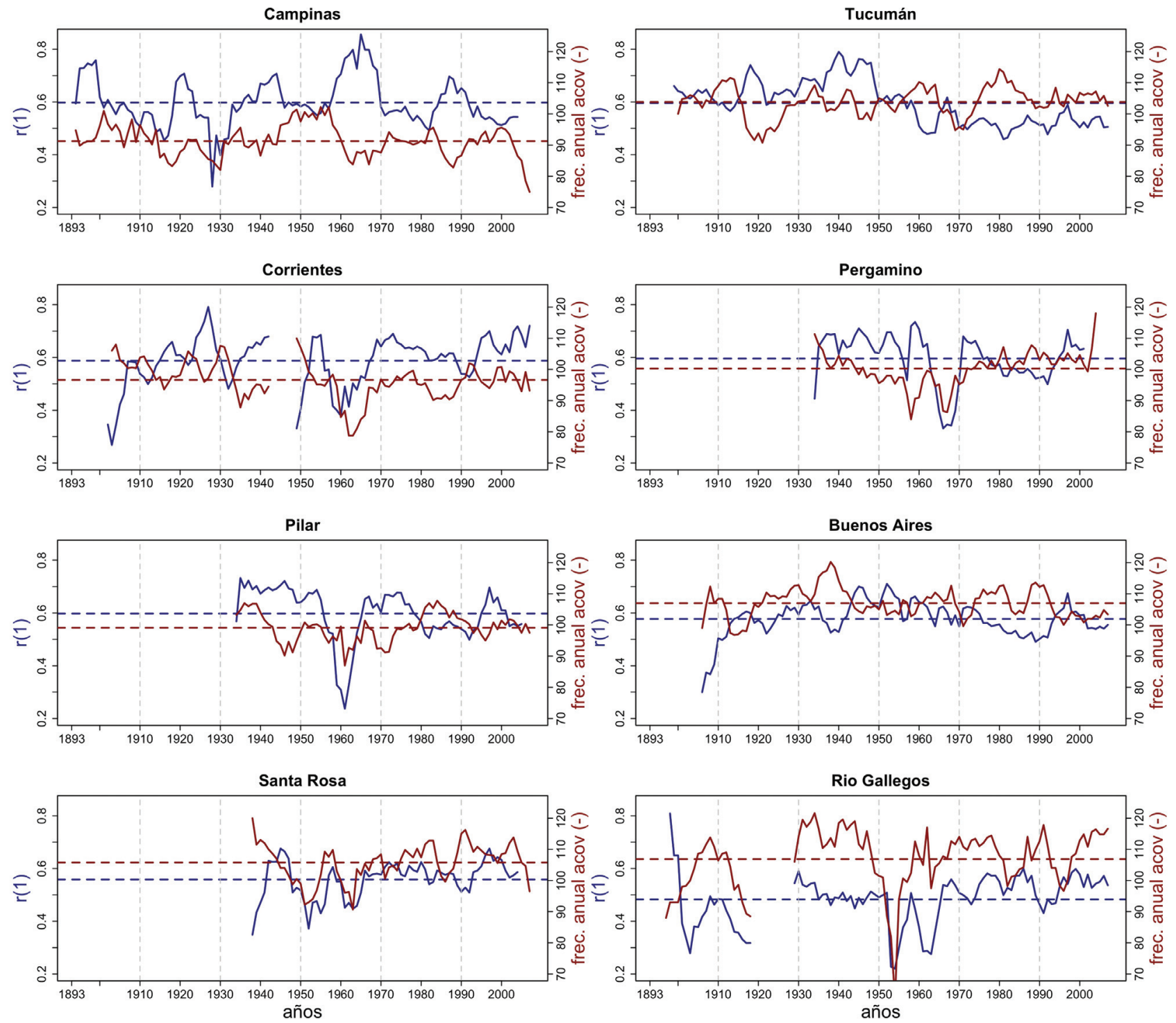

Figura 5 - Marcha interanual del primer coeficiente de autocorrelación calculado según Ec. (11) (azul), y valor medio (azul en línea de puntos) y frecuencia anual de autocovarianzas negativas para el desfase 1 (antipersistencia) en rojo (valor medio en línea de puntos roja) para la temperatura máxima en las estaciones de referencia.

extremos durante este período, seguidos por un incremento de la frecuencia durante los últimos años del siglo veinte (Tencer y Rusticucci, 2012). En este sentido, Rusticucci y Tencer (2008) también reportan cambios en los valores de retorno de los extremos de temperatura debidos principalmente a un salto climático del año 1976-1977. Estos cambios se observan en la forma de una mayor frecuencia de valores altos de temperatura mínima junto con la menor frecuencia de valores altos de temperatura máxima conllevando a una disminución del rango anual de temperatura.

El análisis espectral Wavelet (Fig. 7) para la estimación siguiendo la Ec. (11) muestra la aparición de oscilaciones de períodos menores (entre 8 y 16 años) en contraste con el método descripto por la Ec. (6). La temperatura máxima en Tucumán y Buenos Aires y ambas temperaturas en Río Gallegos presentan máximos de energía centrados en 8 años durante el período 1950-1970. La temperatura mínima en Campinas muestra los mismos patrones para ambos métodos con la única diferencia referida a la presencia de periodicidades de menor período (8-12 años) para el caso en que se considera la media muestral entre 1910-1940. En este sentido, Barrucand y Rusticucci (2001) destacan la evidencia de una importante disminución de la frecuencia de temperaturas mínimas frías de verano que comenzó a finales de la década del 70 y un aumento de la frecuencia de temperaturas máximas cálidas en invierno durante la última década de 1990.

En general, es posible destacar que existen diferencias en la estimación de periodicidades para ambos métodos observándose ondas de mayor período para la estimación donde se analizan las propiedades de las persistencias interna (Ec. (6)). Esto estaría marcando la tendencia de la 

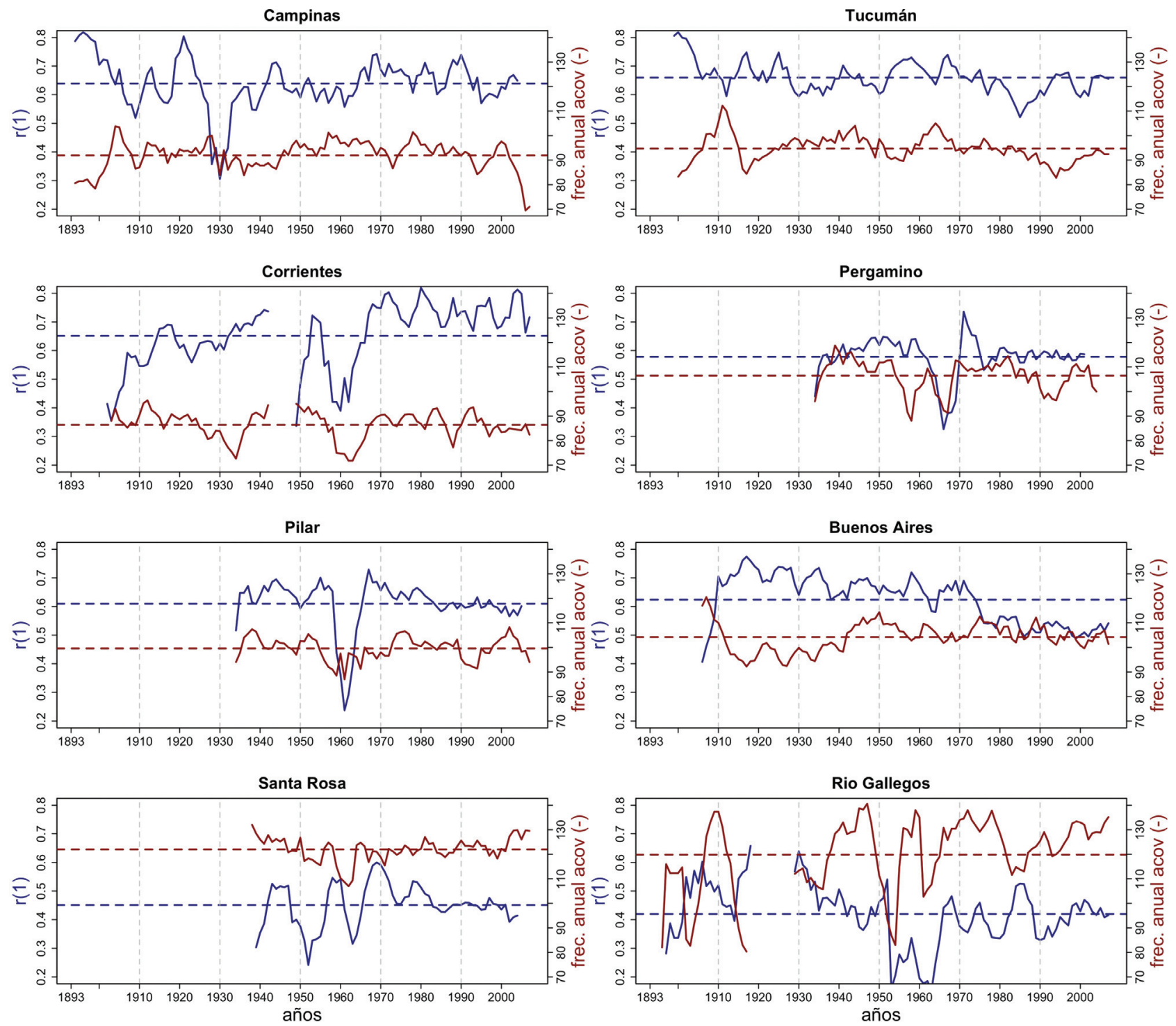

Figura 6 - Marcha interanual del primer coeficiente de autocorrelación calculado según Ec. (11) (azul), y valor medio (azul en línea de puntos) y frecuencia anual de autocovarianzas negativas para el desfase 1 (antipersistencia) en rojo (valor medio en línea de puntos roja) para la temperatura mínima en las estaciones de referencia.

atmósfera en las estaciones analizadas a permanecer en un modo de variabilidad (por ejemplo, mayor cantidad de cambios de estado) independientemente del valor medio de la temperatura.

\section{Conclusiones}

En este trabajo se analizaron las variables que definen la persistencia y la antipersistencia sobre las series de temperatura máxima y mínima diaria en ocho estaciones centenarias en el sudeste de Sudamérica. Se presentan dos metodologías para estimar la función de autocorrelación. La principal diferencia entre estas estimaciones es el uso de medias para cada muestra, que permite comparar la variabilidad interna anual de la persistencia, mientras que para el caso en que se utiliza la media total para referir los resultados anuales respecto a un sistema de referencia común.

En general, se observaron oscilaciones en escalas de tiempo características, desde la escala interanual hasta la decadal. Algunas bandas del espectro presentan características comunes en términos de frecuencias desde la escala interanual (fluctuaciones de entre 4-8 años) hasta escala decadal (12-20 años) y algunas de largo plazo (32-64 años). En general, estas periodicidades presentan cambios con el tiempo, especialmente entre los años 1950-1970. La excepción es Río Gallegos donde la periodicidad de 18 años se observa durante todo el registro.

La persistencia definida mediante el primer coeficiente de autocorrelación es función de la cantidad de términos de autocovarianza negativas. No obstante durante algunos años es dependiente de la magnitud de los términos 


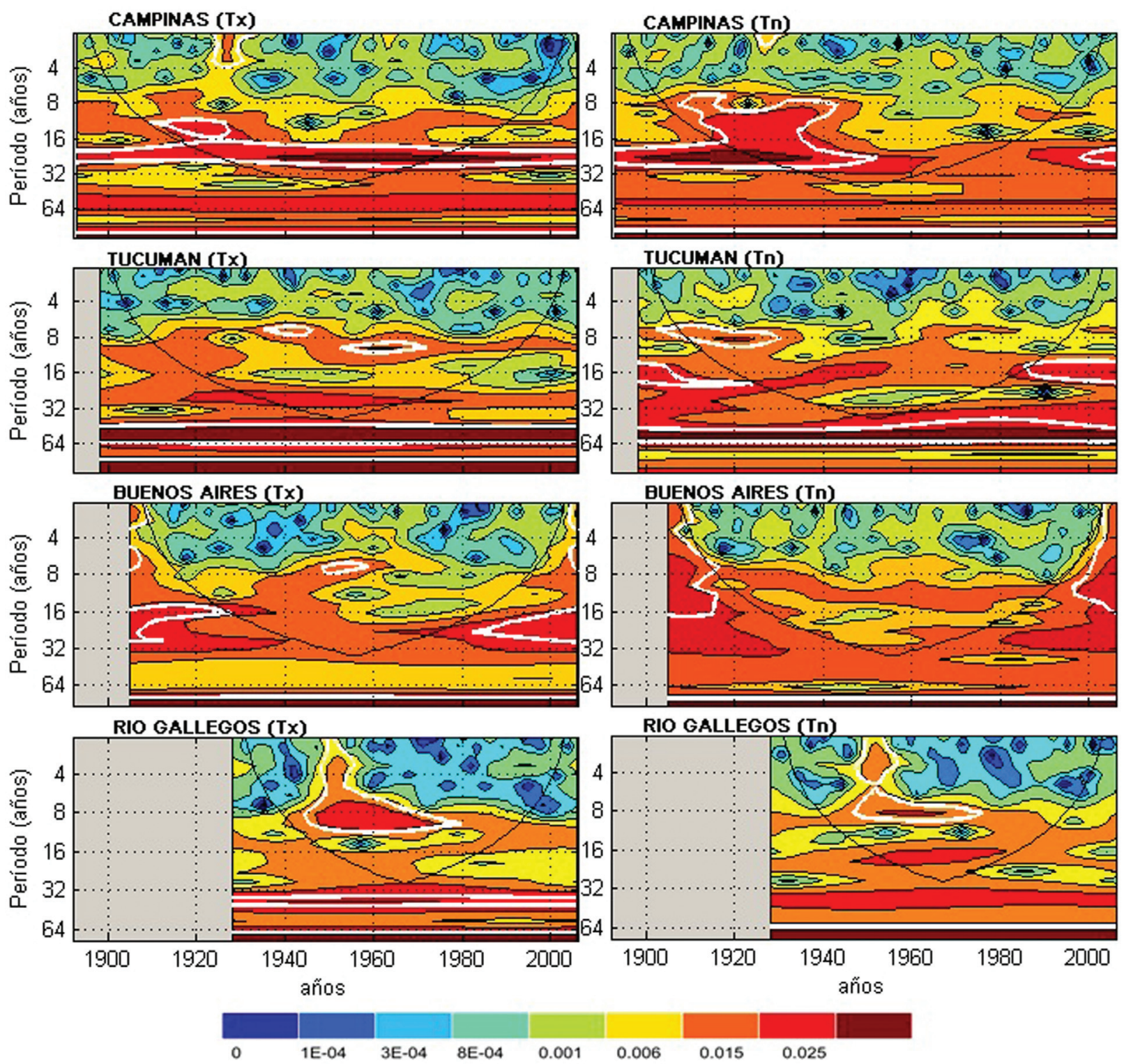

Figura 7 - Espectro Wavelet (adimensional) estimado en base a las marchas interanuales del primer coeficiente de autocorrelación calculado según Ec. (11). Los contornos blancos representan el nivel de significancia del 5\%, respecto de un modelo autoregresivo.

positivos dados por las irrupciones cálidas o frías intensas. Entre los años 1950 y 1970 es posible apreciar un aumento de la variabilidad en estas propiedades.

En términos generales puede inferirse que la cantidad de términos negativos de la autocovarianza es inversamente proporcional a la persistencia. En términos de un análisis sinóptico, el pasaje de frentes y su frecuencia determinan el valor de la persistencia, aunque como se mencionó existen excepciones dadas por la ocurrencia de eventos extremos.

Estos cambios analizados en la persistencia sugieren una variación en la frecuencia de distintos tipos de circulación que tienen un impacto directo en la estructura térmica regional. Así mismo, variaciones de este tipo pueden tener serios impactos socio-económicos ya que estos afectarían directamente la frecuencia y duración de los eventos extremos.

Por esto el seguimiento o monitoreo de los cambios de estado en escala intraanual de las variables que definen tanto a la persistencia como a la antipersistencia ( $r_{1} \mathrm{y}$ cantidad de autocovarianzas negativas) se vuelve una herramienta necesaria a la hora de decidir sobre la validez y comprensión de las características generales del modelo de pronóstico objetivo a utilizar. Además, esto tiene importancia también en el diseño de métodos de control y consistencia ya que en ellos es posible considerar como "outlier" o información errónea los cambios producidos por las irrupciones de escala sinóptica mencionadas anteriormente. 


\section{Agradecimientos}

El presente trabajo fue financiado por el proyecto CONICET PIP 2014-16 11220130100806. Los autores desean agradecer a los revisores anónimos y al editor por sus valiosos comentarios y crítica constructiva.

\section{Referencias}

BARRUCAND, M.; RUSTICUCCI, M. Climatología de temperaturas extremas en la Argentina. Variabilidad temporal y regional. Meteorologica, v. 26, p. 85-102, 2001.

COMPAGNUCCI, E.R.; VARGAS, W.M. Análisis espectral de las series de precipitación estival. Meteorologica, v. 34, p. 213-224, 1983.

DAUBECHIES, I. The wavelet transform time-frequency localization and signal analysis. IEEE Trans. Inform. Theory, v. 36, p. 961-1004, 1990.

FARGE, M. Wavelet transforms and their applications to turbulence. Annu. Rev. Fluid Mech., v. 24, p. 395-457, 1992.

GABRIEL, K.R.; NEUMANN, J. A Markov chain model for daily rainfall occurrence at Tel Aviv. Q.J.R. Meteorol. Soc., v. 88, n. 375, p. 90-95, 1962.

HASSELMANN, K. Stochastic climate models part I. Theory. Tellus A, v. 28, n. 6, p 473-485, 1976.

MADDEN, R.A.; SHEA D.J. Estimates of the natural variability of time-averaged temperatures over the United States. Mon. Wea. Rev., v. 106, p. 1695-1703, 1978.

MINETTI J.L.; CARLETTO, M.C. Estructura espectral de las precipitaciones en ambos lados de la Cordillera de los Andes en Chile y Argentina. Rev. De Geofisica, v. 46, p. 65-74, 1990.

MINETTI, J.L.; VARGAS, W.M. Interaction processes between the annual wave and the disturbances in series of daily temperature. J. of Climate, v. 10, p. 297-305, 1997.

NAUMANN, G.; VARGAS, W.M. Changes in the predictability of the daily thermal structure in southern South America using information theory. Geophysical Research Letters, v. 36, n. 9, p. 1-5, 2009.

NAUMANN, G.; VARGAS, W.M.; MINETTI, J.L. Persistence and long-term memories of daily maximum and minimum temperatures in southern South America. Theoretical and applied climatology, v. 105, n. 3-4, p. 341-355, 2011.

NAUMANN, G.; VARGAS, W.M. A study of intraseasonal temperature variability in southeastern South America. Journal of Climate, v. 25, p. 5892-5903, 2012.

PERCIVAL D.B.; WALDEN A.T. Wavelet methods for time series analysis. Cambridge University Press, Cambridge, 2000 .
RUSTICUCCI, M.; VARGAS, W.M. Las temperaturas de bulbo seco y húmedo en la Argentina. Valores medios y persistencia de anomalías. Meteorologica, v. 18, p. 39-47, 1993.

RUSTICUCCI, M.; VARGAS, W.M. Synoptic situations related to spells of extreme temperatures over Argentina. Meteorological Applications, v. 2, p. 291-300, 1995.

RUSTICUCCI, M.; BARRUCAND, M.G. Climatología de temperaturas extremas en la Argentina. Consistencia de datos. Relación entre la temperatura media estacional y la ocurrencia de días extremos. Meteorologica, v. 26, p. 69-84, 2001.

RUSTICUCCI, M.; VARGAS, W.M. Interannual variability of temperature spells over Argentina. Atmosfera, v. 14, p. 75-86, 2001.

RUSTICUCCI M.; TENCER, B. Observed changes in return values of annual temperature extremes over Argentina. J. Climate, v. 21, p. 5455-5467, 2008.

SHUKLA, J. Predictability in the midst of chaos: A scientific basis for climate forecasting. Science, v. 282, p. 728-731, 1998.

TENCER, B.; RUSTICUCCI, M. Analysis of interdecadal variability of temperature extreme events in Argentina applying EVT. Atmósfera, v. 25, n. 4, p. 327-337, 2012.

TORRENCE, C.; COMPO G.P. A practical guide to Wavelet analysis. Bull. Amer. Meteor. Soc., v. 79, p. 61-78, 1998

TRENBERTH, K. Some effects of finite sample size and persistence on meteorological statistics. Part I: Autocorrelations. Mon Wea Rev., v. 112, p. 2359-2368, 1984a.

TRENBERTH, K. Some effects of finite sample size and persistence on meteorological statistics. Part II: Potential predictability. Mon Wea Rev., v. 112, p. 2369-2379, 1984b.

VARGAS, W.M.; MINETTI, J.L.; POBLETE, A.G. Lowfrequency oscillations in climatic and hydrological variables in southern South America's tropical-subtropical regions. Theor. Appl. Climatol., v. 72, p. 29-40, 2002.

VARGAS, W.M.; NAUMANN, G. Impacts of climatic change and low frequency variability in reference series on daily maximum and minimum temperature in southern South America. Regional Environmental Change, v. 8, p. 45-57, 2008.

WILKS, D.S. Statistical methods in the atmospheric sciences. Elsevier Academic Press, 467 p, 2002.

WORLD METEOROLOGICAL ORGANIZATION (WMO). Guide to climatological practices, fourth edition. WMO n. 100. WMO: Geneva, Switzerland, 2002.

This is an Open Access article distributed under the terms of the Creative Commons Attribution Non-Commercial License which permits unrestricted non-commercial use, distribution, and reproduction in any medium provided the original work is properly cited. 